\title{
NUCLEOSYNTHESIS IN ONEMG NOVAE: COMPARISON WITH OBSERVATIONS
}

\author{
S. WANAJO ${ }^{1}, \mathrm{~K}$. NOMOTO ${ }^{2}$, AND M. HASHIMOTO ${ }^{3}$ \\ ${ }^{1}$ National Astronomical Observatory, Japan \\ 2 University of Tokyo, Japan \\ ${ }^{3}$ Kyushu University, Japan
}

Recent observations show that $\sim 30 \%$ of well-studied novae are classified to $\mathrm{ONeMg}$ novae, occurrences of outbursts with underlying $\mathrm{ONeMg}$ white dwarfs. There is a serious disagreement on the masses of ejected shells between observations and current theory. The estimated ejecta masses for QU Vul, V838 Her, and V1974 Cyg are $\sim 10^{-4}-10^{-3} M_{\odot}$ (Saizar et al. 1992; Shore et al. 1993; Vanlandingham et al. 1996), 10-100 times larger than theoretical estimates (Politano et al. 1995).

We have investigated the nucleosynthesis on $\mathrm{ONeMg}$ novae in wide range of white dwarf and envelope masses by a quasi-analytic method, using an up-to-date nuclear reaction network. Comparison of the nucleosynthetic results with observational abundance estimates enables us to constrain these two parameters. Our results suggest that at least the above three novae have obtained $\sim 10^{-4} M_{\odot}$ of the envelope masses, in good agreement with observational estimates. In addition, the masses of their underlying $\mathrm{ONeMg}$ white dwarfs may have been $\sim 1.1 M_{\odot}$, smaller than previously expected (see Wanajo, Hashimoto, \& Nomoto 1997 for more detail).

\section{References}

Politano, M., Starrfield, S., Truran, J. W., Weiss, A., \& Sparks, W. M. 1995, ApJ, 448, 807

Saizar, P., Starrfield, S., Ferland, G. J., Wagner, R. M., Truran, J. W., Kenyon, S. J., Sparks, W. M., Williams, R. E., \& Stryker, L. L. 1992, ApJ, 398, 651

Shore, S. N., Sonneborn, G., Starrfield, S., Gonzalez-Riestra, R., \& Ake, T. B. 1993, AJ, 106, 2408

Vanlandingham, K. M., Starrfield, S., Wagner, R. M., Shore, S. N., \& Sonneborn, G. 1996, MNRAS, 282, 563

Wanajo, S., Hashimoto, \& Nomoto 1997 , in preparation 